\title{
Markovian SIR model for opinion propagation
}

\author{
E. De Cuypere, K. De Turck, S. Wittevrongel and D. Fiems \\ Department of Telecommunications and Information Processing, Ghent University, \\ St-Pietersnieuwstraat 41, B-9000 Gent, Belgium
}

\begin{abstract}
In this work, we propose a new model for the dynamics of a single opinion propagation at a size-limited location with a low population turnover. This means that a maximum number of individuals can be supported by the location and that the allowed individuals have a long sojourn time before leaving the location. The individuals can have either no opinion (S), a (strong) opinion that they want to spread (I), or an opinion that they keep for themselves (R) (the letters stem from the popular SusceptibleInfectious-Recovered (SIR) epidemic model). Furthermore, we consider a variable opinion transmission rate. Hence, the opinion spreading is modeled as a Markovian nonstandard SIR epidemic model with stochastic arrivals, departures, infections and recoveries.

We apply a numerical approach to this specific SIR epidemic model which rely on a Maclaurin series expansion in order to evaluate the system performance. Finally, we illustrate our approach by some numerical examples.
\end{abstract}

\section{INTRODUCTION}

Given the rapid growth of companies in the internet sector that base their revenue model on advertisement (such as Google, Facebook, etc.) and the ascent of social networks in particular, the study of opinion spreading is a trending topic, and there is a very strong interest in understanding how new opinions spread through a community. In this paper, we study opinion spreading at a specific location. This can be understood as either a virtual location, as on a group page in a social network, or a physical location, for example at an airport where mobile users interact and receive personalized ads.

During the last decades, the main approach for mod- elling such opinion spreading models is the contagion approach, which is based on the spreading of a disease. Most models for the transmission of infectious diseases descend from the classical SIR model [10]. SIR is an abbreviation for susceptible (S), infectious (I) and recovered $(\mathrm{R})$. These are consequently the only possible states that an individual can belong to when we discuss SIR type diseases, and the possible transitions between these states follow $\mathrm{S} \rightarrow \mathrm{I} \rightarrow \mathrm{R}$. In particular, this model suggests that if a healthy individual will encounter a sick individual, there is a specific probability that a healthy individual will get infected and there is a specific probability that an infected individual will get recovered from the disease. Opinion spreading can be interpreted as behaving like a disease: if an individual without a specific opinion about a topic (not opinionated individual) will encounter a strongly opinionated individual, the first individual will, with some probability, be also strongly opinionated. Afterwards, strongly opinionated individuals may, with some probability, stop transmitting their opinion to other not opinionated individuals and keep their own opinion.

In the classical stochastic SIR epidemic model, many unrealistic assumptions are made ([1], [3]). For example, one assumes that the population is constant and that the contact rate (also called the infection rate) and the recovery rate are exponentially distributed. As a consequence to this, many modifications of the model have been introduced and analysed in the literature, where different assumptions have been relaxed. In the paper of Britton [3], a general SIR epidemic model 
is used where the traditional contact rate is replaced with a cyclically varying renewal process with $k$ states. In the paper of Clémençon [4], a specific stochastic epidemic model is studied accounting for the effect of contact-tracing on the spread of an infectious disease. In particular, one considers the situation in which individuals identified as infected may contribute to detecting other infectious individuals by providing information related to individuals with whom they have had possibly infectious contacts.

In the present paper, we consider a location area where at most $L$ individuals can be present and where individuals arrive in accordance with a Poisson process with parameter $\lambda_{\ell}$, for each type of individual $\ell$ in the SIR model. Individuals remain at the location for an exponentially distributed amount of time with mean $1 / \mu$ and then leave. Furthermore, the probability for not opinionated individuals to become strongly opinionated depends on the number of the different individual types present at the location. In particular, the higher the relative number of individuals that want to transmit their opinion, the higher the probability that an individual without an opinion will get one. We also assume that, the higher the number of not opinionated individuals, the higher the probability that one of them gets a (strong) opinion. Concerning the recovery rate, we assume that each strongly opinionated individual has a fixed probability to become opinionated and also that, the more strongly opinionated individuals there are, the higher the probability that one of them becomes opinionated. Further explanation and the motivation on the above assumptions is given in section II. For ease of understanding, not opinionated (S), strongly opinionated (I) and opinionated individuals (R) are further described as susceptible, infected and recovered individuals, respectively.

Also, this paper investigates approximations for the defined SIR epidemic queueing system. In particular, we propose a numerical evaluation method for this
Markovian epidemic queueing system which relies on a Maclaurin-series expansion of the steady-state probability vector. For an overview on the technique of series expansions in stochastic systems, which is also known under the names light traffic analysis or stochastic perturbation, we refer the reader to the survey in [2]. One of the methods to establish series expansions of stochastic models is given via an updating formula in [9]. In [6], cases for which numerical computation of the steady-state vector is possible through a Maclaurin expansion in a parameter $\mu$ are given and the approach is illustrated by a practical example of a paired queueing system.

Further, we also derive the fluid limit of the Markov chain. Fluid limits are a popular mathematical technique (see e.g. [7], [8]) which (when a good scaling is found) allow to focus on the salient features of the stochastic process while discarding 'second-order fluctuations' around this main trend. In the present paper, it helps to make the link with more standard deterministic SIR models. Also, the fluid scaling applied for this model (arrival rates and location capacity are sent to infinity), is highly different and complementary to the Taylor-expansions limit (which holds for low departure rates $\mu$ ). We thus aim to view this difficultto-analyze Markov model from different limiting cases, and gain new insights by combining them. We also note that the derivation of the fluid limit as performed in this paper also lends itself naturally to refinements in the form of diffusion results, but this is considered to be outside of the scope of the current paper.

The remainder of this paper is organised as follows. In section II, the model is described and special cases are given. Next, in section ?? the numerical series expansion approach applied to the opinion propagation system at hand. In section IV we derive a formally justified fluid model from the original Markov model. To illustrate both approaches, section $\mathrm{V}$ considers various numerical examples. Finally, conclusions are drawn in section VI. 


\section{MARKOV CHAIN MODEL}

As arrivals of the individuals are modelled by Poisson processes and the lifetime distribution is exponential, the state of the system can be described by a vector $\mathbf{i}=\left(i_{1}, i_{2}, i_{3}\right) \in \mathcal{L}$ where $i_{1}, i_{2}$ and $i_{3}$ are respectively equal to the number of recovered, infected and susceptible individuals. We denote $\mathcal{L}=$ $\left\{\left(i_{1}, i_{2}, i_{3}\right) \in \mathbb{N}^{3} \mid s(\mathbf{i}) \leq L\right\}$ as the state space of this Markov chain and the sum of all the individuals at the location $s(i)=\sum_{\ell=1}^{3} i_{\ell} \leq L$, must be smaller or equal to the maximum number of individuals $L$. Let $\pi(\mathbf{i})$ denote the steady-state probability distribution of the Markov chain which satisfies the following balance equations,

$$
\begin{gathered}
\pi(\mathbf{i})\left(\sum_{\ell=1}^{3} \mathbb{1}_{\left\{i_{\ell}>0\right\}} \mu s(i)+\sum_{\ell=1}^{3} \mathbb{1}_{\{s(i)<L\}} \lambda_{\ell}\right. \\
\left.+\mathbb{1}_{\left\{i_{3}>0\right\}} \alpha_{s i}\left(i_{1}, i_{2}, i_{3}\right)+\mathbb{1}_{\left\{i_{2}>0\right\}} \alpha_{i r}\left(i_{2}\right)\right) \\
=\sum_{\ell=1}^{3} \pi\left(\mathbf{i}_{\text {up }, \ell}\right) \mu s\left(\mathbf{i}_{\text {up }, \ell}\right) \mathbb{1}_{\{s(i)<L\}} \\
+\sum_{\ell=1}^{3} \pi\left(\mathbf{i}_{\text {down }, \ell}\right) \lambda_{\ell} \mathbb{1}_{\left\{i_{\ell}>0\right\}} \\
+\pi\left(i_{1}, i_{2}-1, i_{3}+1\right) \alpha_{s i}\left(i_{1}, i_{2}, i_{3}\right) \mathbb{1}_{\left\{\left(i_{1}, i_{2}-1, i_{3}+1\right) \epsilon \mathcal{L}\right\}} \\
+\pi\left(i_{1}-1, i_{2}+1, i_{3}\right) \alpha_{i r}\left(i_{2}\right) \mathbb{1}_{\left\{\left(i_{1}-1, i_{2}+1, i_{3}\right) \epsilon \mathcal{L}\right\}} . \quad(1)
\end{gathered}
$$

Indeed, as previously mentioned, we assume the general state-dependent infection rates captured in the function $\alpha_{s i}\left(i_{1}, i_{2}, i_{3}\right)$, and recovery rates which depend on the number of infected users $i_{2}$ only (captured in the function $\alpha_{i r}\left(i_{2}\right)$. Finally, the notation $\mathbf{i}_{\text {up }, \ell}$ and $\mathbf{i}_{\text {down }, \ell}$ describe respectively the increase and the decrease of the $\ell$ th element of the vector $\mathbf{i}$ by one.

\section{Special case 1}

If we assume that each susceptible and infected individual have respectively a constant to get infected and recovered, i.e. $\alpha_{s i}\left(i_{1}, i_{2}, i_{3}\right)=i_{3} \alpha_{s i}$ and $\alpha_{i r}\left(i_{2}\right)=$ $i_{2} \alpha_{s i}$, then the mean number of each type in steady state can be calculated explicitly. Indeed, as the departure rates of each individual are equal to $\mu$, the total number of individuals is distributed as a classic $M / M / L / L$ queue, with arrival rate $\lambda=\lambda_{1}+\lambda_{2}+\lambda_{3}$ and departure rate $\mu$, for which the steady state distribution (denoted as $\operatorname{Pr}[Q=k]$ ) can be found in every queueing-theory textbook. We then compute the stationary fractions of each type $p_{1}, p_{2}$ and $p_{3}$ as follows:

$$
\begin{array}{r}
p_{1}=\frac{\lambda_{1}}{\lambda}+\frac{\lambda_{2}}{\lambda} \frac{\alpha_{i r}}{\alpha_{i r}+\mu}+\frac{\lambda_{3}}{\lambda} \frac{\alpha_{i r} \alpha_{s i}}{\left(\alpha_{i r}+\mu\right)\left(\alpha_{s i}+\mu\right)}, \\
p_{2}=\frac{\lambda_{2}}{\lambda} \frac{\mu}{\alpha_{i r}+\mu}+\frac{\lambda_{3}}{\lambda} \frac{\mu \alpha_{s i}}{\left(\alpha_{i r}+\mu\right)\left(\alpha_{s i}+\mu\right)}, \\
p_{3}=\frac{\lambda_{3}}{\lambda} \frac{\mu}{\alpha_{s i}+\mu},
\end{array}
$$

Indeed, the fraction $p_{3}$ of susceptible individuals is given by the fraction of arriving susceptible users multiplied by the fraction of time during which it has turned into one of the other types. It can be checked that $p_{1}+p_{2}+$ $p_{3}=1$. As we know the distribution of the total number of individuals by the Erlang loss formula (and denote this distribution as $\operatorname{Pr}[Q=k]$ ): we can then compute the distribution of the number of individuals of a certain type $\ell \in\{1, \ldots, 3\}$ as follows:

$$
\operatorname{Pr}\left[Q_{\ell}=n\right]=\sum_{k=n}^{L}\left(\begin{array}{l}
k \\
n
\end{array}\right) \operatorname{Pr}[Q=k] p_{\ell}^{n}\left(1-p_{\ell}\right)^{k-n},
$$

from which the means can be easily computed.

\section{Special case 2}

In the numerical examples and for the fluid model, we often consider the following special case for the functions $\alpha_{s i}\left(i_{3}, i_{2}, i_{1}\right)$ and $\alpha_{i r}\left(i_{2}\right)$ :

$$
\begin{gathered}
\alpha_{s i}\left(i_{1}, i_{2}, i_{3}\right)=\left(\alpha_{s i}^{0}+\alpha_{s i}^{1} \frac{i_{2}}{i_{1}+i_{2}+i_{3}}\right) i_{3}, \\
\alpha_{i r}\left(i_{2}\right)=\alpha_{i r}^{0} i_{2} .
\end{gathered}
$$

where $\alpha_{s i}^{0}, \alpha_{s i}^{1}$ and $\alpha_{i r}^{0}$ are given (positive) rates. The motivation behind this special case is as follows. Firstly, we assume that the higher the relative number of infected individuals at the location, the higher the rate at which the infection spreads. In the case of opinion spreading, the rate that a not opinionated individual gets 
an opinion increases as the number of individuals that want to transmit their opinion, i.e. strongly opinionated individuals, increases. Secondly, we also add a constant term $\alpha_{s i}^{0}$ so that susceptible individuals may become infected by themselves (i.e. without the presence of other infected individuals). Finally, we assume that infected individuals may eventually get recovered: a strongly opinionated individual may, after a while, stop transmitting his opinion and keep it for himself with rate $\alpha_{i r} C_{I R}$.

While the former system of equations is easily solved with a low maximum number of individuals, the state space explodes for even a reasonable $L$ and a direct solution is computationally infeasible.

\section{MACLAURIN-SERIES EXPANSIONS}

?? To mitigate the state space explosion problem, we rely on a Maclaurin series expansion in $\mu$. If $\pi(\mathbf{i})$ is analytic in $\mu=0$, it admits the representation,

$$
\pi(\mathbf{i})=\sum_{n=0}^{\infty} \pi_{n}(\mathbf{i}) \mu^{n},
$$

for $0 \leq \mu<\mu_{0}$ and for $\mathbf{i} \in \mathcal{C}$.

Substituting the former expression in the balance equations yields,

$$
\begin{aligned}
& \sum_{n=0}^{\infty} \pi_{n}(\mathbf{i}) \mu^{n}\left(\sum_{\ell=1}^{K} \mathbb{1}_{\left\{i_{\ell}>0\right\}} \mu s(i)+\sum_{\ell=1}^{K} \mathbb{1}_{\{s(i)<L\}} \lambda_{\ell}\right. \\
& \left.+\mathbb{1}_{\left\{i_{3}>0\right\}} \alpha_{s i}\left(i_{1}, i_{2}, i_{3}\right)+\mathbb{1}_{\left\{i_{2}>0\right\}} \alpha_{i r}\left(i_{2}\right)\right) \\
& =\sum_{n=0}^{\infty} \sum_{\ell=1}^{K} \pi_{n}\left(\mathbf{i}_{\text {up }, \ell}\right) \mu^{n+1} s\left(\mathbf{i}_{\text {up }, \ell}\right) \mathbb{1}_{\{s(i)<L\}} \\
& +\sum_{n=0}^{\infty} \sum_{\ell=1}^{K} \pi_{n}\left(\mathbf{i}_{\text {down }, \ell}\right) \lambda_{\ell} \mu^{n} \mathbb{1}_{\left\{i_{\ell}>0\right\}} \\
& +\sum_{n=0}^{\infty} \pi_{n}\left(i_{1}, i_{2}-1, i_{3}+1\right) \alpha_{s i}\left(i_{1}, i_{2}, i_{3}\right) \mu^{n} \mathbb{1}_{\left\{\left(i_{1}, i_{2}-1, i_{3}+1\right) \epsilon \mathcal{L}\right\}} \\
& +\sum_{n=0}^{\infty} \pi_{n}\left(i_{1}-1, i_{2}+1, i_{3}\right) \alpha_{i r}\left(i_{2}\right) \mu^{n} \mathbb{1}_{\left\{\left(i_{1}-1, i_{2}+1, i_{3}\right) \epsilon \mathcal{L}\right\}} .
\end{aligned}
$$

For $\mathbf{i} \in \mathcal{L}^{*}=\mathcal{L} \backslash\{[L, 0,0]\}$, comparing the terms in $\mu^{0}$ on both sides of the former equation yields,

$$
\pi_{0}(\mathbf{i})=0
$$

whereas comparing the terms in $\mu^{n}$ for $n>0$ gives,

$$
\begin{aligned}
& \pi_{n}(\mathbf{i})= \\
& \frac{1}{\sum_{\ell=1}^{K} \mathbb{1}_{\{s(i)<L\}} \lambda_{\ell}+\mathbb{1}_{\left\{i_{3}>0\right\}} \alpha_{s i}\left(i_{1}, i_{2}, i_{3}\right)+\mathbb{1}_{\left\{i_{2}>0\right\}} \alpha_{i r}\left(i_{2}\right)} \\
& \left(\sum_{\ell=1}^{K} \pi_{n-1}\left(\mathbf{i}_{\text {up }, \ell}\right) s\left(\mathbf{i}_{\text {up }, \ell}\right) \mathbb{1}_{\{s(i)<L\}}\right. \\
& +\sum_{\ell=0}^{K} \pi_{n}\left(\mathbf{i}_{\text {down }, \ell}\right) \lambda_{\ell} \mathbb{1}_{\left\{i_{\ell}>0\right\}} \\
& +\sum_{n=0}^{\infty} \pi_{n}\left(i_{1}-1, i_{2}+1, i_{3}\right) \alpha_{i r}\left(i_{2}\right) \mathbb{1}_{\left\{\left(i_{1}-1, i_{2}+1, i_{3}\right) \in \mathcal{L}\right\}} \\
& \left.-1, i_{3}+1\right) \alpha_{s i}\left(i_{1}, i_{2}, i_{3}\right) \mathbb{1}_{\left\{\left(i_{1}, i_{2}-1, i_{3}+1\right) \epsilon \mathcal{L}\right\}} \\
& \left.-\mathbb{1}_{\{n>0\}} \pi_{n-1}(\mathbf{i}) \sum_{\ell=1}^{K} s(i) \mathbb{1}_{\left\{i_{\ell}>0\right\}}\right) . \quad(6)
\end{aligned}
$$

As detailed in [6], we can use the above equation to compute new terms very efficiently, by iterating over the state space in lexicographic fashion, as on the RHS only entries of either order $n-1$ or entries of order $n$ but with a lower lexicographic index are present.

\section{A. Performance measures}

Once the series expansions of the steady state distribution has been obtained, the expansion of various performance measures directly follows. Let $\mathbf{X} \sim \pi$, then for a performance measure $J=\mathrm{E}[f(\mathbf{X})]$ we have,

$$
\begin{aligned}
J=\sum_{\mathbf{i} \in \mathcal{L}} f(\mathbf{i}) \pi(\mathbf{i})=\sum_{\mathbf{i} \in \mathcal{L}} f(\mathbf{i}) \sum_{n=0}^{\infty} \pi_{n}(\mathbf{i}) \mu^{n} \\
=\sum_{n=0}^{\infty} \sum_{\mathbf{i} \in \mathcal{L}} f(\mathbf{i}) \pi_{n}(\mathbf{i}) \mu^{n}=\sum_{n=0}^{\infty} J_{n} \mu^{n},
\end{aligned}
$$

for $0 \leq \mu<\mu_{0}$ with,

$$
J_{n}=\sum_{\mathbf{i} \in \mathcal{L}} f(\mathbf{i}) \pi_{n}(\mathbf{i}) .
$$

The interchange of the summations is justified by the finiteness of $\mathcal{L}$ and the convergence of $\sum_{n} \pi_{n}(\mathbf{i}) \mu^{n}$ for all $\mathbf{i} \in \mathcal{L}$. As such, any term $J_{n}$ in the expansion of 
a performance measure $J$ can be calculated from the corresponding vector $\pi_{n}$ of the expansion of the steadystate vector. Performance measures of interest include amongst others the $k$ th order moment of the queue content of the $\ell$ th queue $\left(f(\mathbf{i})=i_{\ell}^{k}\right)$.

\section{B. Computational complexity}

As the calculation of $\pi_{n}(\mathbf{i})$ is linear in the size of the state space $S=|\mathcal{L}|$, we have that calculating the first $M$ orders of the expansion takes a time of the order $O(K S M)$.

As the size of the state space is very large, limiting memory consumption is equally important. We note that we only need to store just one vector which has the size of the state space. Assuming one is mainly interested in the expansion of a number of performance measures, note that once the $m$ th term of the expansion of the steady state vector is determined, the corresponding terms in the expansions of various performance measures can be determined as well; see (7). Hence, there is no need to keep track of previous terms of the expansion of steady-state probabilities unless they are required for further calculations of coefficients of steady state probabilities. From (6) one sees that $\pi_{n}(\mathbf{i})$ is expressed in terms of $\pi_{n-1}(\mathbf{j})$, with $\mathbf{j}$ larger than $\mathbf{i}$ (lexicographically). This means that the coefficients of the vector $\pi_{n-1}$ can be overwritten progressively during the calculation of $\pi_{n}$ and memory for only one vector of size $M$ is needed.

\section{FLUID LIMIT}

In this section, we develop a fluid limit for the model described in this contribution. To this end, we make use of the versatile random time-change formulation as proposed by Ethier and Kurtz in their book [7].

Basically, our task consists of two steps: first we write down a time-change formulation of the Markov process at hand, and then we use a strong law of large numbers for Poisson processes so as to transform the stochastic process into a differential equation. For completeness, we state this results here as a lemma.

Lemma 1. Let $Y$ be a unit rate Poisson process. Then for any $U>0$,

$$
\lim _{N \rightarrow \infty} \sup _{0 \leq u \leq U}\left|N^{-1} Y(N u)-u\right|=0 .
$$

Let $\left\{X_{r}^{N}(t), X_{i}^{N}(t), X_{s}^{N}(t)\right\}$ denote a threedimensional continuous-time Markov process, the three components respectively denoting the number of susceptible, infected and recovered users at time $t$, where $N$ denotes the scaling parameter, which affects the system in the following way: arrival rates of the three types are scaled by $N\left(\lambda_{*} \mapsto N \cdot \lambda_{*}\right)$, and the capacity $L$ of the system is likewise scaled with $N$ : $L \mapsto N \cdot L$. We consider in this section both finite and infinite capacity systems, in the latter case of course no capacity scaling is needed. For notational convenience, we introduce $X^{N}(t)=X_{r}^{N}(t)+X_{i}^{N}(t)+X_{s}^{N}(t)$.

The number of susceptible users present at time $t$ consists of: (1) the number of susceptible users present at time $t=0$, which we denote as $X_{s}^{N}(0)$; (2) susceptible users arriving during the interval $(0, t]$ with rate $N \lambda_{3}$; (3) minus the susceptible users that get infected (with a rate that depends on all three processes $X_{s}^{N}$, $\left.X_{i}^{N}, X_{r}^{N}\right)$; (4) lastly, we need to subtract the users that have left the system due to service completion.

We formulate this in terms of a number of independent unit rate Poisson processes $Y_{* *}$ as follows, where the subscripts denote the source and destination of the process at hand with $s, i, r, o$, denoting susceptible, infected, recovered and outside respectively. This manner of writing Markov processes in terms of Poisson processes evaluated at times which dependent on the past of said Markov process (aptly denoted as the random time-change method in [7]) is very handy when it comes to smoothly establishing the corresponding fluid models. 
We have that

$$
\begin{gathered}
X_{s}^{N}(t)=X_{s}^{N}(0)+Y_{o s}\left(N \lambda_{3} \int_{0}^{t} \mathbb{1}_{\left\{X^{N}(\tau)<N \cdot L\right\}} d \tau\right) \\
-Y_{s i}\left(\int_{0}^{t} \alpha_{s i}^{N}\left(X_{s}^{N}(\tau), X_{i}^{N}(\tau), X_{r}^{N}(\tau)\right) \mathbb{1}_{\left\{X_{i}^{N}(\tau)<N \cdot L\right\}} d \tau\right) \\
-Y_{s o}\left(\mu \int_{0}^{t} X_{s}^{N}(\tau) d \tau\right) .
\end{gathered}
$$

Likewise, for the process of number of infected users, we have at time $t$ : (1) users present at time 0 ; (2) arrivals of infected users in $(0, t]$ (when there is room); (3) susceptible users getting infected. Departures occur because of (4) infected users recovering and (5) infected users leaving the system.

$$
\begin{gathered}
X_{i}^{N}(t)=X_{i}^{N}(0)+Y_{o i}\left(N \lambda_{2} \int_{0}^{t} \mathbb{1}_{\left\{X^{N}(\tau)<N \cdot L\right\}} d \tau\right) \\
+Y_{s i}\left(\int_{0}^{t} \alpha_{i}^{N}\left(X_{s}^{N}(\tau), X_{i}^{N}(\tau), X_{r}^{N}(\tau)\right) d \tau\right) \\
-Y_{i r}\left(\int_{0}^{t} \alpha_{i r}^{N}\left(X_{s}^{N}(\tau), X_{i}^{N}(\tau), X_{r}^{N}(\tau)\right) d \tau\right) \\
-Y_{i o}\left(\mu \int_{0}^{t} X_{i}^{N}(\tau) d \tau\right) .
\end{gathered}
$$

And for the process of the number of recovered users:

$$
\begin{gathered}
X_{r}^{N}(t)=X_{r}^{N}(0)+Y_{\text {or }}\left(N \lambda_{1} \int_{0}^{t} \mathbb{1}_{\left\{X^{N}(\tau)<N \cdot L\right\}} d \tau\right) \\
+Y_{\text {ir }}\left(\int_{0}^{t} \alpha_{i r}^{N}\left(X_{s}^{N}(\tau), X_{i}^{N}(\tau), X_{r}^{N}(\tau)\right) d \tau\right) \\
-Y_{\text {ro }}\left(\mu \int_{0}^{t} X_{r}^{N}(\tau) d \tau\right) .
\end{gathered}
$$

Next, we introduce the scaled processes $\bar{X}_{s}:=$ $N^{-1} X_{s}^{N}, \bar{X}_{i}:=N^{-1} X_{i}^{N}, \bar{X}_{r}:=N^{-1} X_{r}^{N}$ and $\bar{X}:=$ $N^{-1} X^{N}$. For the state-dependent infection and recovery rates $\alpha_{i r}$ (and $\alpha_{s i}^{N}$ ), we assume that asymptotically as $N \rightarrow \infty$,

$$
N^{-1} \alpha_{i r}^{N}\left(N i_{1}, N i_{2}, N i_{3}\right) \rightarrow \bar{\alpha}_{i r}\left(i_{1}, i_{2}, i_{3}\right),
$$

and likewise for $\alpha_{s i}$. It can be easily checked that both special cases of section II agree with the above assumption.
Furthermore, we assume that $N^{-1} X_{*}^{N}(0)$ converges to the deterministic constant $x_{*}(0)$ as $N \rightarrow \infty$ (for $*$ equal to $r, i, s)$.

If we introduce the scaled processes in the equations for $X_{*}^{N}(t)$, then we get:

$$
\begin{gathered}
\bar{X}_{s}(t)=x_{s}(0)+N^{-1} Y_{o s}\left(N \lambda_{3} \int_{0}^{t} \mathbb{1}_{\{\bar{X}(\tau)<L\}} d \tau\right) \\
-N^{-1} Y_{s i}\left(N \int_{0}^{t} \bar{\alpha}_{s i}\left(\bar{X}_{s}(\tau), \bar{X}_{i}(\tau), \bar{X}_{r}(\tau)\right) d \tau\right) \\
-N^{-1} Y_{s o}\left(N \mu \int_{0}^{t} \bar{X}_{s}(\tau) d \tau\right) \cdot(11) \\
\bar{X}_{i}(t)=x_{i}(0)+N^{-1} Y_{o i}\left(N \lambda_{2} \int_{0}^{t} \mathbb{1}_{\{\bar{X}(\tau)<L\}} d \tau\right) \\
+N^{-1} Y_{s i}\left(N \int_{0}^{t} \bar{\alpha}_{s i}\left(\bar{X}_{s}(\tau), \bar{X}_{i}(\tau), \bar{X}_{r}(\tau)\right) d \tau\right) \\
-N^{-1} Y_{i r}\left(N \int_{0}^{t} \bar{\alpha}_{i r}\left(\bar{X}_{s}(\tau), \bar{X}_{i}(\tau), \bar{X}_{r}(\tau)\right) d \tau\right) \\
-N^{-1} Y_{i o}\left(N \mu \int_{0}^{t} \bar{X}_{i}(\tau) d \tau\right) .
\end{gathered}
$$

$$
\begin{gathered}
\bar{X}_{r}(t)=x_{r}(0)+N^{-1} Y_{o r}\left(N \lambda_{1} \int_{0}^{t} \mathbb{1}_{\{\bar{X}(\tau)<L\}} d \tau\right) \\
+N^{-1} Y_{i r}\left(N \int_{0}^{t} \bar{\alpha}_{i r}\left(\bar{X}_{s}(\tau), \bar{X}_{i}(\tau), \bar{X}_{r}(\tau)\right) d \tau\right) \\
-N^{-1} Y_{i o}\left(N \mu \int_{0}^{t} \bar{X}_{r}(\tau) d \tau\right) .
\end{gathered}
$$

As every term is either deterministic or of the form $N^{-1} Y_{*}(N \cdots)$, we can apply Lemma 1 , and state that in the limit the process $\left(\bar{X}_{s}(t), \bar{X}_{i}(t), \bar{X}_{r}(t)\right.$ converges to a deterministic limit $\left(x_{s}(t), x_{i}(t), x_{r}(t)\right)$ (with $x(t):=$ $\left.x_{s}(t)+x_{i}(t)+x_{r}(t)\right)$ satisfying the following integral 
equations:

$$
\begin{gathered}
x_{s}(t)=x_{s}(0)+\lambda_{3} \int_{0}^{t} \mathbb{1}_{\{x(\tau)<L\}} d \tau-\int_{0}^{t} \bar{\alpha}_{s i}\left(x_{s}(\tau), x_{i}(\tau)\right. \\
-\mu \int_{0}^{t} x_{s}(\tau) d \tau \\
x_{i}(t)=x_{i}(0)+\lambda_{2} \int_{0}^{t} \mathbb{1}_{\{x(\tau)<L\}} d \tau+\int_{0}^{t} \bar{\alpha}_{s i}\left(x_{s}(\tau), x_{i}(\tau), x_{i}\right. \\
-\int_{0}^{t} \bar{\alpha}_{i r}\left(x_{s}(\tau), x_{i}(\tau), x_{r}(\tau)\right) d \tau-\mu \int_{0}^{t} x_{i}(\tau) d \tau ; \\
x_{r}(t)=x_{r}(0)+\lambda_{1} \int_{0}^{t} \mathbb{1}_{\{x(\tau)<L\}} d \tau+\int_{0}^{t} \bar{\alpha}_{i r}\left(x_{s}(\tau), x_{i}(\tau), x\right. \\
-\mu \int_{0}^{t} x_{r}(\tau) d \tau,
\end{gathered}
$$

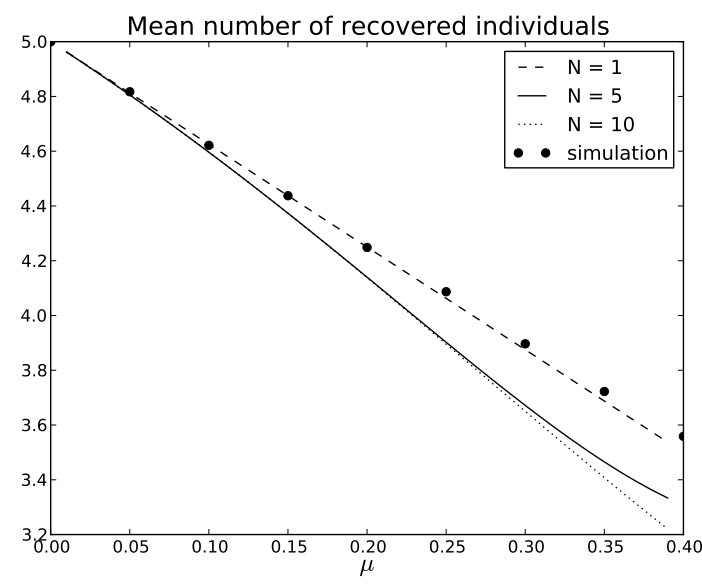

which in turn can be formulated as a system of (nonlinear) differential equations:

$$
\begin{gathered}
\dot{x}_{s}(t)=\lambda_{3} \mathbb{1}_{\{x(t)<L\}}-\bar{\alpha}_{s i}\left(x_{s}(t), x_{i}(t), x_{r}(t)\right)-\mu x_{s}(t) ; \\
\dot{x}_{i}(t)=\lambda_{2} \mathbb{1}_{\{x(t)<L\}}+\bar{\alpha}_{s i}\left(x_{s}(t), x_{i}(t), x_{r}(t)\right. \\
-\bar{\alpha}_{i r}\left(x_{s}(t), x_{i}(t), x_{r}(t)\right)-\mu x_{i}(\tau) ; \\
\dot{x}_{r}(t)=\lambda_{1} \mathbb{1}_{\{x(t)<L\}}+\bar{\alpha}_{i r}\left(x_{s}(t), x_{i}(t), x_{r}(t)\right)-\mu x_{r}(t),
\end{gathered}
$$

This can be solved efficiently with one of the numerous well-honed numerical toolboxes for differential equations. We also note that the equilibrium points (i.e. set $\dot{x}_{*}(t)$ equal to zero in the LHS of the above equations) for special case 2 and $L=\infty$ can be found explicitly as the solutions of a quadratic equation, but we omit the exact expressions due to space constraints.

\section{NUMERICAL RESULTS}

To illustrate our numerical approach, we now assess the accuracy of the perturbation technique by means of some numerical examples. First, consider a system with a maximum number of individuals $L$ equal to 5 . Moreover, the arrival intensity of each type of individual is equal to 1 and we consider the second special case with $\alpha_{i r}^{0}, \alpha_{s i}^{0}$ and $\alpha_{s i}^{1}$ are all equal to 3 . Figure 1 , 2 and 3 depict respectively the mean recovered queue

Fig. 1. Mean number of recovered individuals.

content, the mean infected queue content and the mean susceptible queue content versus the lifetime rate $\mu$. Series expansions of various orders are depicted as indicated $(N=1,5,10)$, as well as simulation results which allow for assessing the accuracy of the series expansions. As expected, the mean number of recovered individuals decreases and the mean number of infected and susceptible individuals increase as the departure rate increases. Moreover, for $\mu=0$, the population consists only of recovered users as their lifetime is infinite such that all individuals get recovered eventually. Also, we observe that the approximation method is fairly accurate for low orders of the expansions (e.g. highly accurate for $N=10$ in figure 3). Finally, for these parameter settings, the mean number of infected and susceptible individuals have a non-linear behavior in $\mu$.

\section{Special case 1}

In contrast to the previous part, we now look at the first special case as described in section II, where the mean number of each individual type are calculated explicitly. Figure 4 and 5 depict the mean number of individuals of type $i$ and $s$ versus the arrival rate of infected individuals $\lambda_{2}$ for different values of the infection rate $\alpha_{s i}$. Moreover, the maximum number of individuals allowed at the location $L$ equals 10 , the 


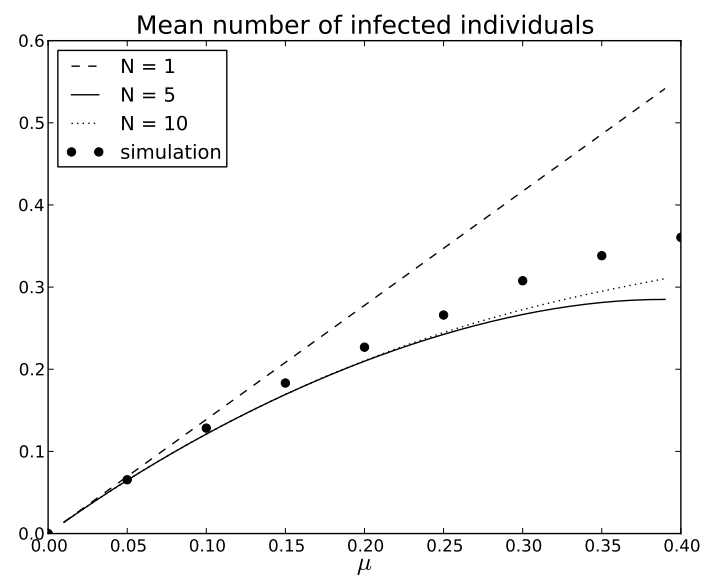

Fig. 2. Mean number of infected individuals.

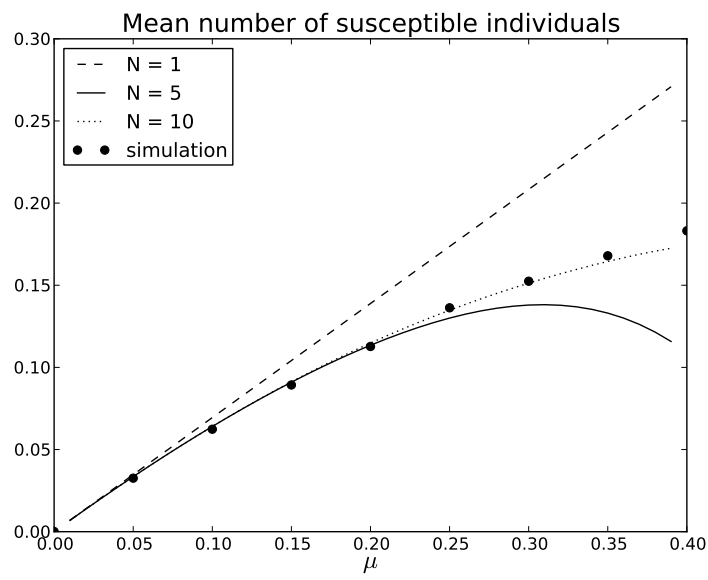

Fig. 3. Mean number of susceptible individuals.

arrival intensity of recovered and susceptible individuals and the recovery rate are equal to 3 , the lifetime rate $\mu$ equals 0.01 and the order of the series expansion $M$ equals 10. As expected, the higher the arrival rate of infected individuals $\lambda_{2}$, the lower the mean number of susceptible individuals and the higher the mean number of infected and recovered individuals. This trend is strengthened when the infection rate $\alpha_{s i}$ increases.

\section{Fluid limit}

We look at the fluid approximation for the second special case in Figs. 6 and 7. In the first plot we start

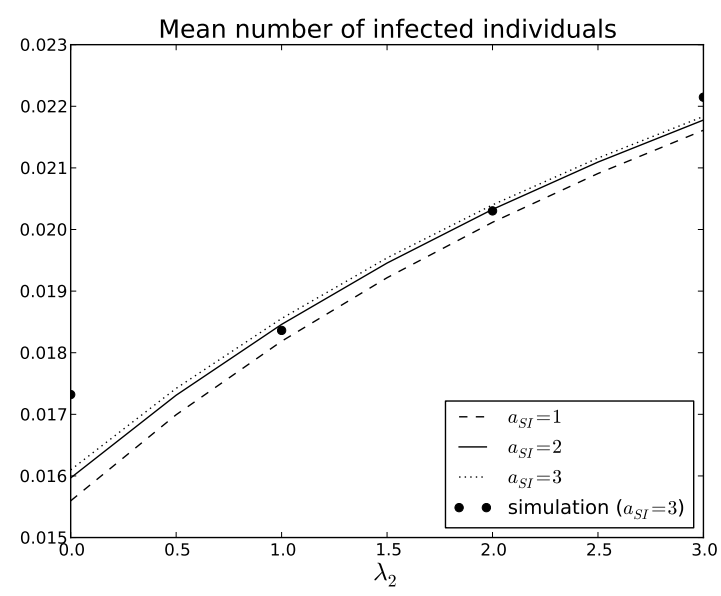

Fig. 4. Mean number of infected individuals.

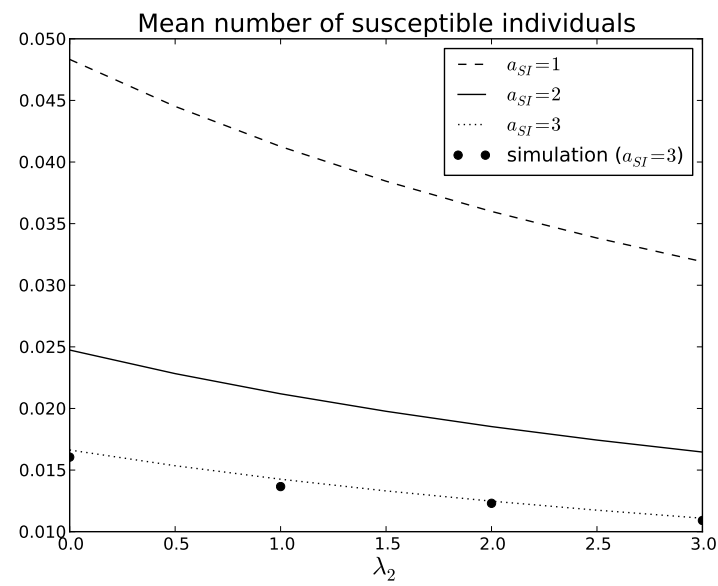

Fig. 5. Mean number of susceptible individuals.

from a large number of infected users which quickly leads to many recovered users, whereas in the second the large number of recovered users prevents a large infected population taking place. We see good correspondence with the simulations.

\section{CONCLUSION}

In this paper, we evaluate the performance of a single opinion propagation in a size-limited location that has a low population turnover. This means that the allowed individuals remain there on average for a long period. Furthermore, we assume that individuals can have either 


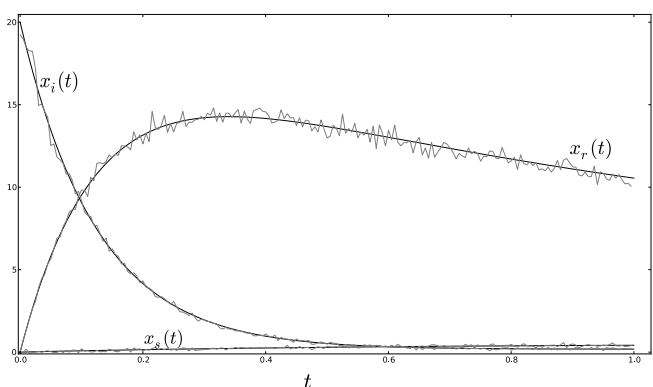

Fig. 6. Fluid model and simulations for $N=100, \lambda_{*}=1, \mu=1$, $\alpha_{*}^{*}=3$.

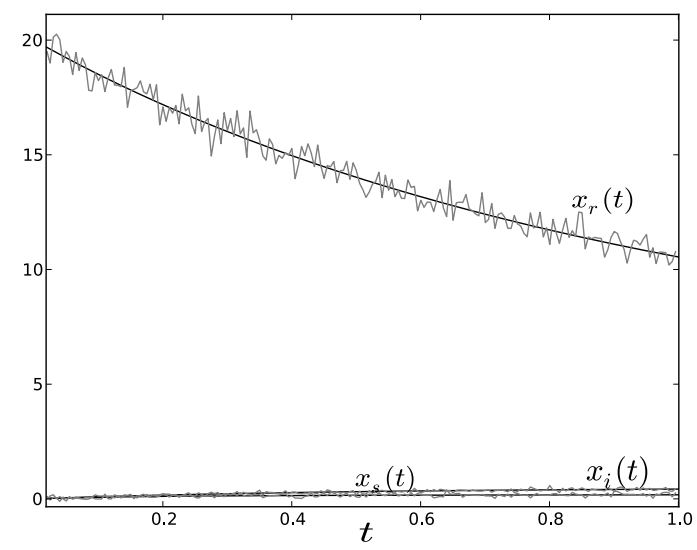

Fig. 7. Fluid model and simulations for $N=100, \lambda_{*}=1, \mu=1$, $\alpha_{*}^{*}=3$.

no opinion (S), a strong opinion that they want to spread (I) or an opinion that they keep for themselves (R). Moreover, the rate at which an individual without an opinion may get an opinion varies according to the number of the different individual types present at the location.

To cope with the inherent state-space explosion, we propose an approximative numerical algorithm of the queueing system at hand. In particular, a numerical algorithm is applied which calculates the first $M$ coefficients of the Maclaurin series expansion of the steady-state probability vector in $O(S M)$ operations, $S$ being the size of the state space. Out of the numerical results we may conclude that the series expansion is quite a good approximation for the opinion model only when the departure rate is small. Future work will focus on expanding the system to a multiple opinion propagation model and deriving diffusion approximations.

\section{REFERENCES}

[1] H. Andersson and T. Britton, Stochastic epidemic models and their statistical analysis, Springer Lecture Notes in Statistics, Springer-Verlag, New York., 151, 2000.

[2] B. Błaszczyszyn, T. Rolski, V. Schmidt. Advances in Queueing: Theory, Methods and Open Problems, chapter Light-traffic approximations in queues and related stochastic models. CRC Press, Boca Raton, Florida, 1995.

[3] T. Britton and M. Lindholm, The early stage behaviour of a stochastic SIR epidemic with term-time forcing, Journal of Applied probability, 46(4), 975-992, 2009.

[4] S. Clémençon, A stochastic SIR model with contact-tracing: large population limits and statistical inference, Journal of Biological Dynamics, 2(4), 392-414, 2008.

[5] E. De Cuypere, D. Fiems, Performance evaluation of a kitting process, Proceedings of the 18th International Conference on Analytical and Stochastic Modelling Techniques and Applications (ASMTA 2011), pp. 175-188, Venice, June 2011.

[6] K. De Turck, E. De Cuypere, S. Wittevrongel and D. Fiems, Algorithmic approach to series expansions around transient Markov chains with applications to paired queuing systems, International Conference on Performance Evaluation Methodologies and Tools (VALUETOOLS 2012), 38-44, France, October 2012.

[7] S.N. Ethier and T.G. Kurtz, Markov Processes: Characterization and Convergence. Wiley and Sons, Second Edition, 2005.

[8] Ph. Robert, Stochastic Networks and Queues. Springer, Berlin, 2003.

[9] B. Heidergott, A. Hordijk, and N. Leder, Series Expansions for Continuous-Time Markov Processes, Oper. Res., 58.3, 756-767, May 2010.

[10] W.O. Kermack and A.G. McKendrick, A Contribution to the Mathematical Theory of Epidemics, Proc. R. Soc. Lond. A, 115, 700-721, 1927.

[11] M. Kitsak and L.K. Gallos and S. Havlin and F. Liljeros and L. Muchnik and H.E. Stanley, Identification of influential spreaders in complex networks, Nature Physics, 6(11), 888-893, 2010. 